
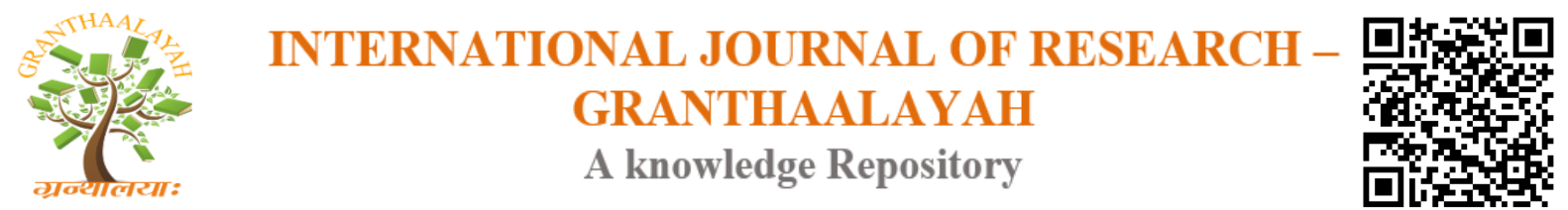

Science

\title{
PLANETARY MAGNETIC FIELD AND GRAVITY IN THE SOLAR SYSTEM
}

\author{
Samir A Hamouda ${ }^{1}$, Eman A. Alsslam Alfadeel ${ }^{2}$, Mohamed Belhasan Mohamed ${ }^{3}$ \\ 1,2,3 Department of Physics, University of Benghazi, Benghazi, Libya
}

\begin{abstract}
Gravity plays a major role in the planetary formation and the development of the solar system. Gravity attraction is the essence of a power that holds and governs the universe; it makes the planets in the solar system revolve around the sun and the moons around their planets. Magnetic fields are also an important phenomenon in the solar system and beyond. Their causes are complex and have a variety of effects on their surroundings; they have become a critical tool for the exploration of solar system bodies. However, the study of the mechanisms of planets formation in the solar system is a difficult problem made more so by the inability to construct planetary-scale models for laboratory study. However, understanding the nature of the matter comprising the Solar System is crucial for understanding the mechanism that generates planetary magnetic fields and planetary gravity. In this study, a brief history about the development of planetary gravity is presented. Some data about the physical properties of planets in the solar system are presented and discussed. However, much work is still needed before the planetary gravity and planetary magnetic field processes are fully understood and full advantage be taken of the implications of both phenomena observations.
\end{abstract}

Keywords: Planetary Gravity; Planetary Magnetic Field; Planets, Magnetism; Solar System; History; Dynamo Theory.

Cite This Article: Samir A Hamouda, Eman A. Alsslam Alfadeel, and Mohamed Belhasan Mohamed. (2017). "PLANETARY MAGNETIC FIELD AND GRAVITY IN THE SOLAR SYSTEM." International Journal of Research - Granthaalayah, 5(9), 145-151. https://doi.org/10.29121/granthaalayah.v5.i9.2017.2224.

\section{Introduction}

Gravity is the attractive force between all objects, but it is most significant in the case of massive bodies. Perhaps, the most striking mechanism about gravity, it sets up a tension of mutual attraction resulting in an order that extends to all matter [1]. Unlike the other known forces in Nature, gravity is very weak but acts over very large length scales even across the universe. The large scale of interaction between bodies is the key to a profound influence of gravity over all things large in the universe [2]. Therefore, studying the gravity of the planetary system can help in understanding our universe. 


\section{Materials and Methods}

There are three great names in the history of man's understanding of gravity: Galileo Galilei, who was the first to study in detail the process of free and restricted fall; Isaac Newton, who first had the idea of gravity as a universal force; and Albert Einstein, who said that gravity is nothing but the curvature of the four-dimensional space-time continuum [3]. However, many variables which must be considered before a reasonable representation about the solar system formation can be formulated.

As stated by [3], that Newton, having obtained the basic ideas of Universal Gravity in the very beginning of his scientific career, it took about twenty years long delay before it was published in 1687. The reason for such a delay is the fact that he lacked the mathematical methods necessary for the development of all the consequences of his fundamental law of interaction between the material bodies.

Newton had to assume that the force of gravity is inversely proportional to the square of the distance between the centers of these two bodies. But when an apple is attracted by the terrestrial globe, the force pulling it down is composed of infinite number of different forces caused by the attraction of rocks at various depths under the roots of the apple tree, by the waters of the Pacific Ocean, and by the molten central iron core of the Earth [3] (see figure1). As a result, Newton had to prove that all these forces add up to a single force which would be present if all the mass of earth were concentrated in its center [3].

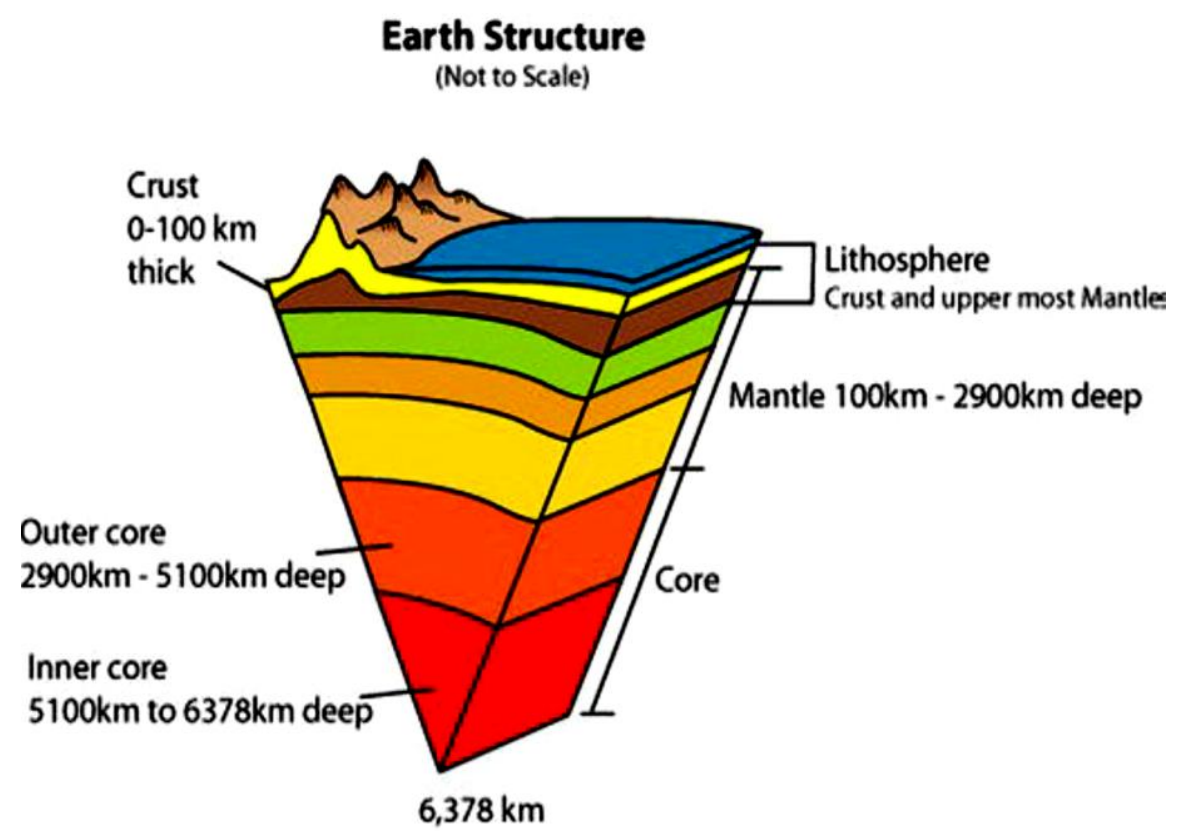

Figure1: Earth's structure [4]

The inner core is solid and has a density of about $15 \mathrm{~g} / \mathrm{cm} 3$. It is believed to be made of a nickeliron alloy ( $\mathrm{Ni}$ about 5-10 wt. \%) with very small amounts of some lighter elements. It is the outcome of the Earth differentiation with the heavier elements sinking towards the center of the 
planet after its formation. It is unattached to the mantle, suspended in the molten outer core and, in spite of its high temperature $(5000-6000 \mathrm{C})$, it is believed to have solified as a result of pressure-freezing which occurs to most liquids under extreme pressure.

The outer core has a composition similar to the inner core with about $10 \%$ of sulphur, oxygen and other light elements. The temperature is $4000-5000 \mathrm{C}$ and the density is between 10 and 12 $\mathrm{g} / \mathrm{cm} 3$. Since the pressure is lower and it is hot, the outer core is liquid. Over geologic time, the inner core grows at the expense of the outer core as the whole Earth cools. The pressure is the same but the temperature drops and the pressure-freezing radius extends farther out. In the outer core the liquid metal is electrically conducting. This conductive layer combines with Earth's rotation to create a dynamo effect that maintains a system of electrical currents that creates the Earth's magnetic field. In conclusion, the Earth's magnetism is due to a combination of rotation and molten metallic core [4]

On the other hand, Einstein's concept of the gravitational field grew from his Special Theory of Relativity, and the Special Theory was based on the theory of the electromagnetic field formulated by James Clerk Maxwell. But in spite of many attempts, Einstein and those who have followed him have failed to establish any contact with Maxwell's electrodynamics [3].

However, it was; Michael Faraday who believed in the existence of a relationship between gravity and electricity, despite the fact that his thought, neither has been derived, one from the other, nor has a common root been found from which they might be generated [11] [3].

Most of the early discoveries into the nature of planetary magnetic fields were made by studying the most accessible magnetized object: the Earth, or the most powerful: the Sun. It has been known since ancient times that magnetized objects responded to some kind of force inherent in the Earth. Chinese legends suggest compasses were used in 2634 BC, and the first recorded use is found in a Chinese text published at the end of the 11th century. Attempts to explain the workings of the phenomenon began with William Gilbert in 1600; he developed a model of a magnetized Earth, in which he believed that the Earth rotated because it was magnetic. An astronomer Heinrich Schwabe, searching in 1826-1843 for the proposed planet Vulcan inside Mercury's orbit, discovered the 11-year sunspot cycle and noted that geomagnetic storms corresponded to sunspot maximums [5][6]. In (1955) Parker gave the fluid dynamo proposal that is essentially the model used today. Although various other mechanisms for generating the geomagnetic field have been proposed, only the dynamo concept is seriously considered today [7].

The dynamo theory is a geophysical theory that explains the origin of Earth's main magnetic field in terms of a self-exciting (or self-sustaining) dynamo. In this dynamo mechanism, fluid motion in Earth's outer core moves conducting material (liquid iron) across an already existing weak magnetic field and generates an electric current. (Heat from radioactive decay in the core is thought to induce the convective motion.) The electric current, in turn, produces a magnetic field that also interacts with the fluid motion to create a secondary magnetic field. Together, the two fields are stronger than the original and lie essentially along the axis of Earth's rotation [8] [9] In the Introduction section, present clearly and briefly the problem investigated, with relevant references. 


\section{Results and Discussions}

It was suggested [10] that the study of the interiors of the various terrestrial planets leads to the possibility of planetary gravity being linked to the electromagnetism generated in the planetary interiors. Such a conclusion implies that Earth's gravitational attraction may be attributed to magnetic coupling experienced between Earth's electromagnetism and all the earthly objectselectrically charged or uncharged. More precisely, terrestrial gravity is deemed to be outcome of the bound state of the planetary electromagnetism [10].

This can be seen from the physical properties of planets in solar system given in table 1 .

Table1: summarizes relevant information for the planets in the solar system [12]

\begin{tabular}{|l|l|l|}
\hline Planet & $\mathbf{g}(\mathbf{m} / \mathbf{s} 2)$ & B(Tesla) \\
\hline EARTH & 9.81036 & $3.10 \mathrm{E}-05$ \\
\hline MERCURY & 3.714552 & $2.00 \mathrm{E}-07$ \\
\hline JUPITER & 25.92916 & $4.25 \mathrm{E}-04$ \\
\hline SATURN & 11.21185 & $2.10 \mathrm{E}-05$ \\
\hline URANUS & 9.021487 & $2.30 \mathrm{E}-05$ \\
\hline NEPTUNE & 11.0004 & $1.40 \mathrm{E}-05$ \\
\hline
\end{tabular}

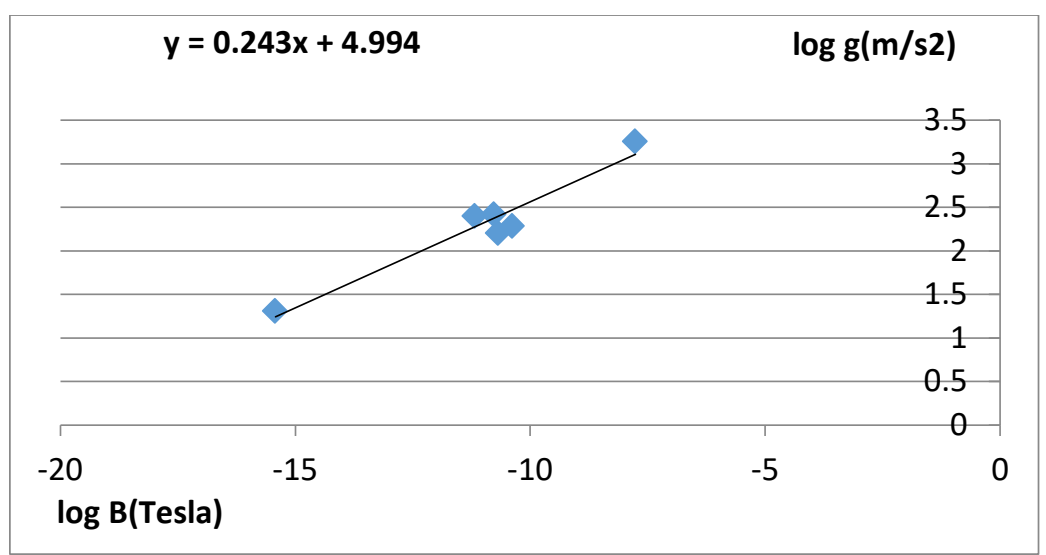

Figure2: Shows the log- log graph for gravity and magnetic field of planets in the solar system

It can be seen from figur2 that there appears to be a link between the Magnetic field and the gravity field of planets in the solar system. This link can be expressed as:

$$
g \propto B^{0.243}
$$

Table 2: gives gravity fields for the planets and distances from the sun [12]

\begin{tabular}{|l|l|l|}
\hline planet & $\mathbf{g}$ & $\mathbf{R}(\mathbf{K m})$ \\
\hline VENUS & 8.868639 & 6052 \\
\hline EARTH & 9.81036 & 6371 \\
\hline MARS & 3.714552 & 3390 \\
\hline JUPITER & 25.92916 & 69911 \\
\hline
\end{tabular}




\begin{tabular}{|l|l|l|}
\hline SATURN & 11.21185 & 58232 \\
\hline URANUS & 9.021487 & 25362 \\
\hline NEPTUNE & 11.0004 & 24624 \\
\hline PLUTO & 0.61683 & 1187 \\
\hline
\end{tabular}

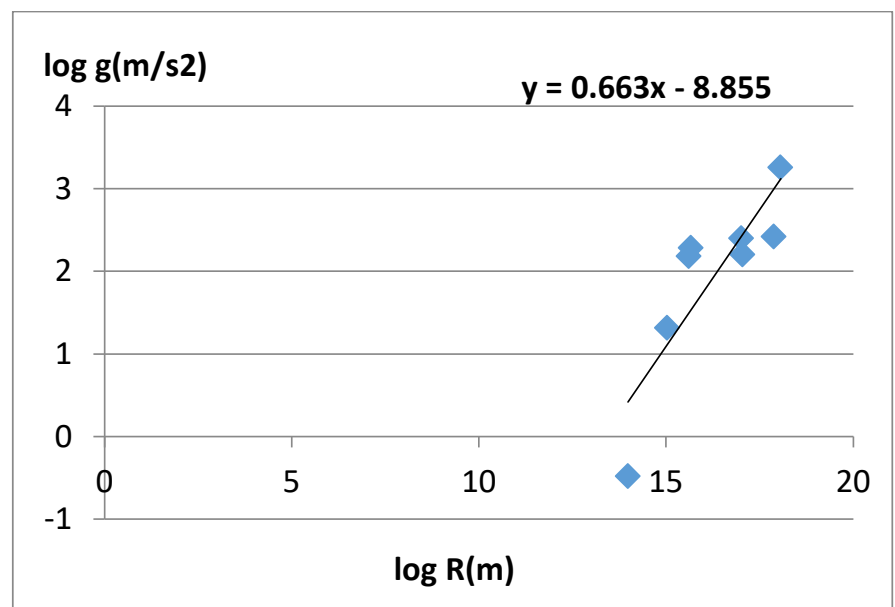

Figure 2: Shows the log- log graph for gravity and distances from the sun for planets in the solar system

It can be seen from figure 3 that there appears to be a link between gravity fields and distances from the sun for the planets in the solar system. This link can be expressed as:

$$
\mathrm{g} \propto \mathrm{R}^{0.663}
$$

Table 3: Gives Centripetal Accelerations and distances from the sun for the planets in the solar system

\begin{tabular}{|l|l|l|l|}
\hline planet & $\boldsymbol{\omega} \mathbf{~ r a d} / \mathbf{s}^{2}$ & $\mathbf{R}(\mathbf{m e t e r})$ & $\mathbf{a c = \boldsymbol { \omega } ^ { 2 } . \mathbf { R }}$ \\
\hline Venus & $3.24 \mathrm{E}-07$ & $1.0472 \mathrm{E}+11$ & 0.010993 \\
\hline Earth & $1.99 \mathrm{E}-07$ & $1.496 \mathrm{E}+11$ & 0.005924 \\
\hline Mars & $1.06 \mathrm{E}-07$ & $2.3936 \mathrm{E}+11$ & 0.002689 \\
\hline Jupiter & $1.68 \mathrm{E}-08$ & $7.7792 \mathrm{E}+11$ & 0.00022 \\
\hline Saturn & $6.76 \mathrm{E}-09$ & $1.496 \mathrm{E}+12$ & $6.84 \mathrm{E}-05$ \\
\hline Uranus & $2.37 \mathrm{E}-09$ & $2.93216 \mathrm{E}+12$ & $1.65 \mathrm{E}-05$ \\
\hline Neptune & $1.21 \mathrm{E}-09$ & $5.80448 \mathrm{E}+12$ & $8.5 \mathrm{E}-06$ \\
\hline Pluto & $8.04 \mathrm{E}-10$ & $1.15491 \mathrm{E}+13$ & $7.47 \mathrm{E}-06$ \\
\hline
\end{tabular}




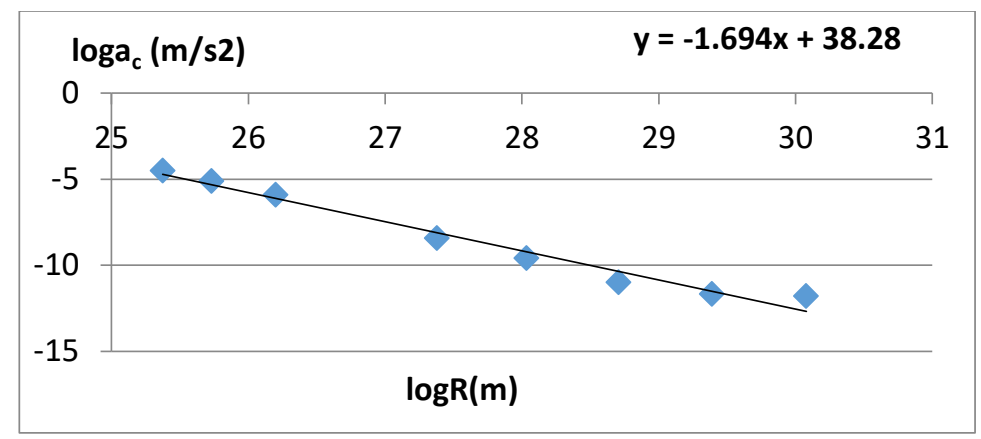

Figure 3: Shows the log-log graph for centripetal accelerations for the planets as a function of distances from the sun.

It can be seen from figure 3 that there appears to be a link between centripetal accelerations and distances from the sun for the planets in the solar system. This link can be expressed as:

$$
a_{c} \propto \frac{1}{R^{1.694}}
$$

\section{Conclusions and Recommendations}

It can be said that mathematics has certainly evolved enough to do a very good job of describing gravity and magnetism. For detailed analysis purposes Newton's law of gravity can be considered as a general law the same as hook's law. When introducing the concept of stress and strain it became clear the physical meaning of the force constant (k). Similar approach should be adapted in order to define the nature of the gravitational constant $(\mathrm{G})$. It was pointed out by Dirac, that the large ratio of electric to gravitational forces is the characteristic for the present stage of our universe. He concluded that the gravitational constant $(\mathrm{G})$ is decreasing with time, and this decrease may be associated with the expansion of the universe due to the steady rarefaction of the material filling it. On the other hand, Edward Teller pointed out that the variation of the gravitational constant $(\mathrm{G})$ would result in the change of temperature. Therefore, the real advances in the collection, analysis and interpretation of planetary gravity and magnetic data studies are currently prevented only by a lack of sufficient data. New surveys to integrate the available data sets and to increase the detail of the information would represent a decisive step to reveal the nature of planetary gravity and magnetic field phenomenon

\section{References}

[1] RONALD E. MICKLE,GRAVITY'S INFLUENCE ON THE DEVELOPMENT OF THE SOLAR SYSTEM, Meteoritics \& Planetary Science 40, Nr 4, (2005)

[2] Marc A. Murison, An Introduction to Gravity in the Solar System,(2005). http://alpheratz.net/papers/SolarSystemGravity/SolarSystemGravity.pdf

[3] George Gamow, Gravity, Science Study Series, Published by Anchor Books with Doubleday \& Company, Inc. Garden City, New York. (1962).

[4] The Magnetic field of the Earth and Planets, http://eureka01.pd.infn.it:5210/ marzari/FISICA_DEL_MEZZO/magnetic.pdf

[5] Richard McDonald, (2005), "Planetary Magnetic Fields". http://www.themcdonalds.net/richard/astro/papers/602-magfields.pdf(General Internet site)

[6] William Gilbert. http://galileo.rice.edu/sci/gilbert.html, (General Internet site) 
[7] Paul Charbonneau, (2005), "Dynamo Models of the Solar Cycle”, Living Rev. Solar Phys., 2. http://solarphysics.livingreviews.org/resources/download/lrsp-2005-2Color.pdf

[8] Ethan, (2011) "Why does the Earth have a liquid core?" Science Blogs. http://scienceblogs.com/startswithabang/2011/09/28/why-does-the-earth-have-a-liqu/

[9] Samir Ahmed Hamouda, Nada Eaz-Alden Emgau, Rabab Muftah Bohagar, Aisha Mohammed Eissa., (2017)., STUDY OF PLANETARY MAGNETIC FIELDS., International Journal of Research - Granthaalayah, 5(3), 29-44. https://doi.org/10.5281/zenodo.439552.

[10] Ashwini Kumar Lal, "On Planetary Electromagnetism and Gravity". International Journal of Astronomy and Astrophysics, 2011, 1, 62-66 http://www.scirp.org/journal/ijaa,

[11] Michael Faraday, (2003), on the various forces of nature. Vigyan Prasar,New Delhi http://www.arvindguptatoys.com/arvindgupta/vp-faraday-nature\%20.pdf

[12] THE SOLAR SYSTEM. http://docplayer.net/13482005-The-solar-system-i-basic-compositionand-structure.html.

*Corresponding author.

E-mail address: dr_s_hamouda@ yahoo.ie 